\title{
INTRUSIONES SOBRE TRASTORNOS ALIMENTARIOS EN POBLACIÓN GENERAL: DESARROLLO Y VALIDACIÓN DEL INVENTARIO DE PENSAMIENTOS INTRUSOS ALIMENTARIOS (INPIAS)
}

\author{
ConXa PerpiñÁ ${ }^{1,2}$, MARÍA Roncero ${ }^{2}$ y AMPARO Belloch ${ }^{2}$ \\ ${ }^{1}$ Ciber Fisiopatología Obesidad y Nutrición (CIBEROBN), Instituto Salud Carlos III, España \\ ${ }^{2}$ Facultad de Psicología, Universidad de Valencia
}

\begin{abstract}
Resumen: El objetivo del presente estudio fue el desarrollo y validación del Inventario de Pensamientos Intrusos Alimentarios (INPIAS), autoinforme que evalúa la frecuencia de intrusiones de contenido alimentario, sus repercusiones emocionales y estrategias de control. Los participantes fueron 358 provinentes de la comunidad. El análisis de componentes principales indicó en la parte primera (tipo/frecuencia intrusiones) una estructura bi-factorial: Intrusiones de descontento normativo e Impulsos de psicopatología alimentaria. La parte 2A (consecuencias, valoraciones) se compuso de dos factores: Carga emocional y Profecía cumplida/significado personal, y la parte 2B (estrategias de control) de 4 factores: Estrategias normalizadoras, Neutralización/supresión, Rituales no alimentarios y No control. La consistencia interna y fiabilidad test-retest fueron excelentes. El INPIAS diferenció entre personas con y sin riesgo de padecer un trastorno alimentario, siendo sus intrusiones cuantitativamente y cualitativamente distintas. El INPIAS es útil en la evaluación de intrusiones relacionadas con los trastornos alimentarios, un área poco estudiada y de creciente interés en el espectro obsesivo-compulsivo.
\end{abstract}

Palabras clave: Intrusiones, trastornos alimentarios, espectro obsesivo-compulsivo, estudio instrumental.

Eating-related intrusions in a community sample: development and validation of the Eating-Related Intrusive Thoughts Inventory (INPIAS)

\begin{abstract}
The purpose of the present study was to develop and validate the Eating-related Intrusive Thoughts Inventory (INPIAS), a self-report that evaluates the frequency of intrusions with an eating-related content, their emotional repercussions and control strategies. Three hundred fifty eight participants from the community took part in this investigation. The principal components analysis of the first part (type and frequency of intrusions) indicated a bi-factorial structure: Normative discontent intrusions and Eating psychopathology impulses. Part 2A (consequences and ratings) was made up of two factors: Emotional charge and Fulfilled prophesy/personal significance. Part 2B (control strategies) consisted of four factors: Normalizing strategies, Neutralization/suppression, Non-eating rituals and No control. The internal consistency and the test-retest reliability were excellent. The INPIAS was able to differentiate between people with and without risk of suffering an eating disorder, as their intrusions were quantitatively and qualitatively different. The INPIAS is useful evaluating intrusions related to eating disorders, an area that has been studied little and is of increasing interest in the obsessive-compulsive spectrum.
\end{abstract}

Keywords: Intrusive thoughts, eating disorders, obsessive-compulsive spectrum, instrumental study.

\section{INTRODUCCIÓN}

Los trastornos alimentarios (TA), o de la conducta alimentaria, se caracterizan por un patrón

Recibido 27 marzo 2008; aceptado 06 mayo 2008

Correspondencia: Conxa Perpiñá Tordera, Departamento de Personalidad, Evaluación y Tratamientos Psicológicos, Facultad de Psicología, Universidad de Valencia, Av. Blasco Ibáñez 21, 46010 Valencia (España). Correo-e: perpinya@uv.es alimentario alterado (restringir la alimentación, tener un impulso irresistible por comer, etc.), junto a una serie de prácticas encaminadas a

Agradecimientos: Esta investigación ha sido financiada en parte por el Ministerio de Educación y Ciencia (SEJ2006/03893-PSIC) y en parte por La Conselleria de Empresa, Universidad y Ciencia Dirección General de Investigación y Transferencia Tecnológica. Generalitat Valenciana (AE/07/022). CIBER Fisiopatología de la Obesidad y Nutrición es una iniciativa de ISCIII. 
«compensar» los efectos de la ingesta (vomitar, tomar laxantes, hacer ejercicio excesivo, etc.).

En esta última década, son muchos los estudios que resaltan la relación que existe entre los TA y los trastornos obsesivos compulsivos (TOC). Ambos trastornos presentan una alta comorbilidad tanto desde un nivel de síntomas (20$30 \%$ ) (Humphreys, Clopton y Reich, 2007), como de síndromes, oscilando en este caso los datos entre el 16\% (Bullick, Sullivan, Fear, y Pickering, 1997) y el 60\%, dependiendo del diagnóstico concreto y sus correspondientes subtipos (Serpell, Livingstone, Neiderman y Lask, 2002). El TOC suele anteceder al TA (y por tanto a la demacración) (Thornton y Russell, 1997), y cuando el peso se restaura, algunas de las características obsesivas siguen estando presentes (Ranson, Kaye, Weltzin, Rao y Matsunaga, 1999). Dichas características son las que se relacionan con lo que se suele calificar como el «sustrato de personalidad del TOC»: el perfeccionismo, la rigidez, la necesidad de orden y simetría, o la constricción emocional, entre otras (Serpell et al., 2002), lo que está llevando a considerarlas como factores de vulnerabilidad y predisposición para el padecimiento de un TA. Desde esta perspectiva, ambas condiciones representarían diferentes aspectos de un hipotético continuo compulsivo-impulsivo, es decir una amplia gama de patologías caracterizadas por pensamientos intrusos, impulsos irresistibles, y rituales de conductas más o menos finalistas (Speranza, et. al., 2001). La conjunción de éstos y otros argumentos ha llevado a considerar la posibilidad de que los TA pudieran ser una variante fenotípica del TOC (Speranza et al., 2001), o bien variantes fenotípicos de la misma diátesis genética (Milos, Sindler, Ruggiero, Klaghofer y Schnyder, 2002).

También desde los planteamientos cognitivos se están comenzando a investigar las coincidencias y similitudes entre los TA y el TOC. Por lo que se refiere al TOC, se ha sugerido que los contenidos de las obsesiones clínicas no difieren de ciertos pensamientos intrusos (PI) análogos a obsesiones que experimentan la gran mayoría de la población general (Belloch, Morillo, Lucero, Cabedo y Carrió, 2004; Clark y Purdon, 1993; Rachman y de Silva, 1978; Belloch, Morillo, García-Soriano, 2006; Gimenez,
Morillo, Belloch, Carrió y Cabedo, 2004). El paso de un PI normal a una obsesión patológica se produciría, según esos planteamientos, por la atribución de un significado disfuncional a la presencia y/o el contenido del PI lo que, a su vez, daría lugar a la puesta en marcha de estrategias activas para su neutralización y/o control. El modelo cognitivo de los TA postula, por su parte, que los pensamientos disfuncionales con respecto al tamaño corporal, la figura, el peso, o lo que se debe comer para conseguir controlar el cuerpo, constituyen no sólo la causa de los síntomas manifiestos de los TA sino que además contribuyen a su mantenimiento o perpetuación (Jones, Harris y Leung, 2005). Así pues, parece razonable suponer que una parte significativa de los comportamientos disfuncionales relacionados con el patrón alimentario anómalo se produzcan como consecuencia de la intrusión repetida y persistente de pensamientos sobre la propia apariencia y el peso, que generarían malestar e incertidumbre y que provocarían la puesta en marcha de comportamientos tales como verificar el propio peso, hacer ejercicio, purgarse, o restringir la ingesta, entre otros. Si bien en el caso de los TA, en términos psicopatológicos, hablamos de ideas sobrevaloradas y en el TOC de obsesiones, lo cierto es que el miedo a ganar peso, hacer dieta, ejercicio, verificar el peso, atracarse y purgarse tienen un carácter intruso y comportan rituales que interfieren y perjudican gravemente la calidad de vida de las personas. Pero además, el estudio del tipo de PI que ocurren en los TA brinda la oportunidad de trabajar con «contenidos» que son normativos y socialmente deseables y aceptados (a diferencia de lo que ocurre con los PI análogos a las obsesiones clínicamente significativas).

En este contexto, el estudio de los denominados PI disfuncionales, que se encuentran en la base de trastornos como el TOC, la depresión, la ansiedad generalizada, el estrés post-traumático y, por supuesto, los TA (Clark, 2005), puede considerarse como un punto de partida sólido sobre el que empezar a comprender los elementos psicopatogénicos comunes y específicos de los trastornos mencionados. Sin embargo, las investigaciones publicadas al respecto son todavía muy escasas. Por otra parte, sería 
muy conveniente el poder contrastar la naturaleza y propiedades de este tipo de intrusiones cognitivas entre la población no clínica y la que recibe un diagnóstico clínico, en el caso del interés del presente estudio, de un TA.

Por tanto, los objetivos del presente trabajo fueron: (1) desarrollar y validar un instrumento de autoinforme capaz de detectar presencia de intrusiones de contenido análogo a las que experimentan las personas con TA (en adelante, pensamientos intrusos alimentarios, PIA) en la población general, y las repercusiones emocionales que la intrusión más molesta tienen en la persona que las experimenta, así como las estrategias de control y/o neutralización que promueven; 2) examinar su estructura factorial, fiabilidad y relaciones con otras variables relevantes en el estudio de los TA y los TOC; y 3) analizar las diferencias entre personas en riesgo de padecer un TA (población subclínica) y personas sin riesgo, en la frecuencia de PIAs, así como en sus consecuencias emocionales y comportamentales (estrategias de control).

\section{MÉTODO}

\section{Participantes}

En esta investigación participaron 358 personas provenientes de la comunidad, (estudiantes de Psicología de la Universidad de Valencia y familiares o amigos de éstos; véase procedimiento). Del total, 267 eran mujeres $(74,6 \%)$ y 91 hombres $(25,4 \%)$, con una media de edad de $23,86(D T=4,05$; rango: $14-38)$. La media de índice de masa corporal (IMC. $\left.\mathrm{kg} /(\mathrm{m})^{2}\right)$ fue de $22,17(D T=3$; rango 17,03-32,87). La mayor parte de la muestra estaba soltera $(85,5 \%)$, tenía un nivel socioeconómico medio $(72,10 \%)$, y el nivel de estudios era predominantemente universitario $(64,30 \%)$.

\section{Instrumentos}

Inventario de Pensamientos Intrusos Alimentarios (INPIAS) ${ }^{1}$. Se trata del cuestionario

\footnotetext{
${ }^{1}$ Se puede pedir una copia del INPIAS a la primera autora
}

objeto de estudio y construido para el análisis de los PIAs en población general. Este autoinforme se ha inspirado en el INPIOS (Inventario de Pensamientos Intrusos Obsesivos) (García-Soriano, 2008), diseñado a su vez para superar algunas de las limitaciones del Revised Obsessional Intrusions Inventory (ROII; Purdon y Clark, 1994), y cuyo propósito es valorar la frecuencia con que la población no clínica experimenta pensamientos, imágenes o impulsos intrusos con contenidos típicamente obsesivos (p.ej., comprobación, limpieza, repetición, etc.), así como las consecuencias emocionales, la interferencia, y las estrategias realizadas para atenuar la molestia generada por tales pensamientos.

Para el contenido de los items del INPIAS se siguió un procedimiento «racional» considerando los contenidos más importantes en la psicopatología de los TA y los formatos de intrusiones (pensamiento, imagen e impulso). Igualmente, también se tuvo en cuenta el contexto en el que esas intrusiones podrían suscitarse (antes, durante o después de comer; al mirarse en un espejo, al estar con los demás, etc.) ${ }^{2}$. En las instrucciones del instrumento se da una breve explicación acerca de lo que significan las intrusiones, enfatizando que se trata de eventos molestos, que interrumpen el pensamiento, y que experimentan todas las personas con mayor o menor frecuencia. El INPIAS consta de dos partes. La primera evalúa a lo largo de 50 items la frecuencia con la que la persona experimenta determinadas intrusiones sobre patrones de ingesta, ejercicio e imagen corporal en una escala de 0 (nunca) a 6 («durante todo el día»). En la segunda parte se evalúa la reacción emo-

\footnotetext{
${ }^{2}$ Dos de las autoras (C.P. y A.B.) elaboraron un listado inicial de 98 enunciados, todos ellos referidos a posibles intrusiones relacionadas con la comida, la dieta, el ejercicio, el peso, la apariencia física, o las conductas compensatorias, agrupadas en diversos contextos, y formulados en términos de pensamiento, imagen o impulso. Una vez eliminadas las duplicidades y corregida la redacción inicial, el listado se redujo a 70 afirmaciones que fueron a su vez objeto de valoración y discusión por los integrantes del grupo más amplio de investigación en el que se encuadra este trabajo, formado en su mayor parte por especialistas en el TOC y los TA. Tras este proceso de evaluación, la primera parte del inventario quedó reducido a 50 items, que son los que finalmente se han considerado para su análisis en este trabajo.
} 
cional, la interferencia, y las valoraciones disfuncionales que ocasiona el PIA considerado por la persona como más molesto y experimentado en los últimos tres meses (16 items) y finalmente, se pide que indique las estrategias de afrontamiento que lleva a cabo con respecto a ese PIA (17 items). Esta segunda parte se puntúan en una escala de 0 (nada, nunca) a 4 (extremadamente, siempre).

The Restraint Scale (RS) -Escala de Restricción- (Herman y Polivy, 1980). Autoinforme que evalúa las fluctuaciones de peso, el grado de restricción y las actitudes hacia el peso y la comida. Se suele utilizar también como medida de cribado para detectar población subclínica y de riesgo de padecimiento de un TA. La puntuación de corte de 16 diferencia entre personas restrictivas y no restrictivas (Heatherton, Herman, Polivy, King y McGree, 1988). Consta de 10 items que se responden en una escala Likert de 4 ó 5 puntos según el ítem. La consistencia interna ( $\alpha$ de Cronbach) en el presente estudio osciló entre 0,83 (Escala total) y 0,70 (subescala de fluctuación del peso»), siendo de 0,79 la obtenida para la subescala «preocupación por la dieta».

The Eating Attitudes Test (EAT-26) - Cuestionario de Actitudes hacia la Comida- (Garner, Olmsted, Bohr y Garfinkel, 1982; versión abreviada del EAT-40 de Garner y Garfinkel, 1979). Para este trabajo se ha empleado la adaptación española realizada por Castro, Toro, Salamero y Guimerá (1991). Evalúa actitudes y comportamientos relacionados con los TA, principalmente con la anorexia nerviosa. Los 26 ítems se contestan en una escala tipo Likert de 6 puntos (desde «nunca» hasta «siempre»). Se obtiene una puntuación total, además de la de tres subescalas. La consistencia interna que se ha obtenido en el presente estudio es de 0,90 para la escala global, 0,89 («Hacer dieta»), 0,76 («Bulimia y preocupación por el alimento») y 0,60 («Control oral»).

Multidimensional Body-Self Relations Questionnaire (MBSRQ-AS34) - Cuestionario de Relaciones Multidimensionales Cuerpo-mí mismo-(Brown, Cash, y Mikulka, 1990). Es una medida de evaluación actitudinal de imagen corporal. En la versión de 34 ítems consta de 5 escalas (Evaluación de la apariencia; Orientación a la apariencia; Satisfacción áreas corporales; Preocupación por el sobre-peso y Auto-clasificación del peso) que se contestan en un formato de respuesta de 5 puntos de acuerdo-desacuerdo. En este estudio, la consistencia interna ha oscilado entre 0,89 y 0,75 según las sub-escalas.

Clark-Beck Obsessive Compulsive Inventory (C-BOCI) - Inventario Obsesivo-compulsivo de Clark y Beck-(Clark y Beck, 2002). Se ha utilizado la adaptación y validación a población española de Reina, Belloch, Morillo y Clark (2007). Es un instrumento de screening para evaluar la frecuencia de síntomas obsesivos y compulsivos. Está compuesto de 25 items en una escala de respuesta tipo Likert de 4 puntos (0: «nunca» - 3: «con mucha frecuencia»). En el presente trabajo se ha obtenido una consistencia interna para la escala total de 0,89 , de 0,85 para la sub-escala de obsesiones y de 0,79 para la de compulsiones.

Inventario de Creencias Obsesivas (ICO) (Belloch, et al., 2003; Cabedo et al., 2004). Autoinfome diseñado para evaluar las diferentes áreas de creencias disfuncionales relacionadas en el desarrollo y/o mantenimiento del TOC. Se compone de 50 items que se contestan en una escala Likert de 7 puntos (1: «Completamente en desacuerdo» - 7: «Completamente de acuerdo»). Los ítems se agrupan en 8 sub-escalas: Responsabilidad excesiva; Importancia de los pensamientos; Fusión Pensamiento Acción (FPA)probabilidad; FPA-moral; Importancia de controlar los pensamientos; Sobreestimación de la amenaza; Intolerancia a la incertidumbre y Perfeccionismo. En el presente trabajo la consistencia interna ha oscilado entre 0,88 y 0,75 según las escalas.

Multidimensional Perfectionism Scale (MPS) -Escala Multidimensional de Perfeccionismo- (Frost, Marten, Lahart y Rosenblate, 1990). Se ha utilizado la versión española realizada por Carrasco (2006). Diseñado para medir distintas dimensiones de perfeccionismo, consta de 35 items con un formato de respuesta de 5 puntos de desacuerdo-acuerdo. Además de una puntuación total, la versión original contiene 6 subescalas: Preocupación sobre los errores, Estándares Personales; Expectativas parentales; Críticas parentales; Dudas de las acciones 
y Organización. La consistencia interna ha oscilado en el presente estudio entre 0,94 y 0,75 , según las escalas.

Penn State Worry Questionnaire (PSWQ) - Cuestionario de preocupación de Pensilvania- (Meyer, Miller, Metzger y Borkovec, 1990). Se ha empleado la versión española de Sandín y Chorot (1991). Evalúa el constructo de «worry» o tendencia general a preocuparse. Consta de 16 ítems en una escala de autoadscripción del ítem de 5 puntos (1:»Nada típico en mí»-5:»Muy típico en mí»). La consistencia interna en este estudio ha sido de 0,74 .

\section{Procedimiento}

Todos los participantes lo fueron de forma voluntaria y dieron su consentimiento por escrito. La captación de los mismos se realizó siguiendo la estrategia de «bola de nieve» que a continuación se especifica. En la Facultad de Psicología de Valencia (curso 2006/2007) se ofreció la posibilidad de participar en una investigación de campo, para lo cual debían asistir a un seminario sobre los TA y los PI a impartir por de una de las autoras (M.R.) y poder mejorar la nota de la asignatura siempre y cuando estuvieran aprobados. Los estudiantes que accedieron, completaron el protocolo de evaluación anteriormente comentado antes de asistir al Seminario. Una vez allí, se les explicó el propósito de cada uno de los instrumentos y de la investigación en general, se aclararon las posibles dudas, y se les adiestró en la administración de los instrumentos a terceros. A continuación, se les pidió que los administraran a 4 familiares o amigos (en edades comprendidas entre los 14-40 años) de forma individual. Además de los límites de edad, como criterios de exclusión se tuvo en cuenta que los participantes no tuvieran historia de trastorno mental, o hubieran recibido tratamiento psicológico o farmacológico durante los dos últimos años, o presentaran dificultad para leer y comprender instrucciones escritas en español. Por algunas de estas razones, o por no haber completado adecuadamente los instrumentos, se excluyeron 36 personas, resultando final- mente los 358 participantes descritos al inicio de este apartado. Todos los participantes cumplimentaron el protocolo que se ha descrito anteriormente tras haber firmado el consentimiento informado. El orden de administración de los cuestionarios se balanceó aleatoriamente, a excepción del INPIAS que siempre aparecía en primer lugar. Además, parte de los participantes $(\mathrm{N}=191)$ volvieron a cumplimentar el INPIAS entre una o dos semanas después con objeto de obtener datos para la estabilidad del instrumento (re-test).

En cuanto al procedimiento estadístico, en el caso de los análisis de contenido del INPIAS se llevaron a cabo diversos análisis factoriales exploratorios. Todos los análisis se ajustaron a los parámetros siguientes: componentes principales; scree test de Cattell (1966), y coeficiente alfa de generalizabilidad de Kaiser (Kline y Barret, 1983), para la retención del número más adecuado de factores en cada caso; punto de corte para la inclusión de items (saturación factorial) $\geq 0,40$; medida de adecuación muestral para los análisis: Kaiser-Meyer-Olkin (KMO). Rotación: oblicua (promax), para examinar la asociación entre factores, seguida, en su caso, de varimax. Para la estimación de los tamaños de las correlaciones entre factores se siguieron los criterios de Cohen (1988), según los cuales: $r \geq 0,50=$ tamaño grande; $r$ entre $0,40-0,30$, tamaño medio; $r$ entre 0,29 0,10 , tamaño pequeño. La consistencia interna se analizó mediante el estadístico de Cronbach, y la estabilidad temporal con el coeficiente de correlación intraclase (CCI). Para los análisis de la validez convergente y discriminante se llevaron a cabo análisis de correlación Pearson. Los análisis de diferencias entre dos grupos se realizaron mediante pruebas $t$ con las correcciones de Levene y las pruebas robustas correspondientes. Para controlar el peso de alguna variable que pudiera estar influyendo en las diferencias de los grupos, se llevaron a cabo análisis de covarianza (ANCOVA), introduciendo como covariables las medidas que en cada caso se supusiera podían estar influyendo en las diferencias observadas. Todos los análisis se realizaron con el paquete estadístico SPSS 14.0 software (SPSS, Chicago, IL, USA). 


\section{RESULTADOS}

Validez de contenido del INPIAS: Estructura Factorial

Se realizaron tres conjuntos de análisis diferenciados, uno para cada parte del cuestionario: INPIAS-Parte 1 (frecuencia de intrusiones sobre patrones y hábitos alimentarios e imagen corporal), INPIAS-Parte 2-A (consecuencias emocionales, interferencia, y valoraciones relacionadas con la intrusión más molesta de entre las listadas en la parte 1), INPIAS-Parte 2B (estrategias de afrontamiento ante la intrusión más molesta). Cada uno de estos conjuntos de análisis se comenta por separado.
- INPIAS Parte 1. Contenidos de intrusiones sobre patrones alimentarios e imagen corporal.

La rotación promax inicial indicó que la estructura más adecuada era bi-factorial. Se observó una correlación de tamaño medio $(r=$ $0,42)$ entre los dos factores extraídos. Sólo 3 items no alcanzaron el nivel de saturación exigido. El primer factor explicó una varianza del $24,77 \%$ y en el segundo la varianza explicada fue del $19,57 \%$. Teniendo en cuenta estos primeros resultados, se aplicó una rotación ortogonal (varimax; $\mathrm{KMO}=0,961)$ que mostró la misma estructura y que incluía los 3 items excluidos con el procedimiento anterior. En consecuencia se

Tabla 1. Análisis factorial exploratorio (pesos factoriales), INPIAS-Parte 1:

Contenido y frecuencia de intrusiones $(N=358)$

\begin{tabular}{|c|c|c|c|}
\hline Ítem & $\begin{array}{c}\text { Factor } 1 \\
\text { Descontexto normativo }\end{array}$ & Ítem & $\begin{array}{c}\text { Factor } 2 \\
\text { Impulsos psicopatología alimentaria }\end{array}$ \\
\hline $\begin{array}{r}29 \\
28 \\
32 \\
44 \\
19 \\
9 \\
49 \\
50 \\
31 \\
46 \\
7 \\
30 \\
16 \\
41 \\
2 \\
25 \\
39 \\
37 \\
21 \\
27 \\
13 \\
38 \\
1 \\
18 \\
8 \\
14 \\
40 \\
15 \\
33 \\
45 \\
35 \\
42 \\
34 \\
43 \\
24 \\
5 \\
4 \\
3 \\
\end{array}$ & $\begin{array}{l}0,79 \\
0,78 \\
0,77 \\
0,76 \\
0,76 \\
0,75 \\
0,74 \\
0,74 \\
0,74 \\
0,73 \\
0,73 \\
0,73 \\
0,73 \\
0,72 \\
0,71 \\
0,70 \\
0,70 \\
0,69 \\
0,69 \\
0,67 \\
0,66 \\
0,66 \\
0,64 \\
0,64 \\
0,64 \\
0,64 \\
0,62 \\
0,61 \\
0,59 \\
0,59 \\
0,55 \\
0,55 \\
0,53 \\
0,51 \\
0,50 \\
0,41 \\
0,38 \\
0,35\end{array}$ & $\begin{array}{r}22 \\
17 \\
6 \\
36 \\
47 \\
20 \\
12 \\
23 \\
26 \\
11 \\
48 \\
10\end{array}$ & $\begin{array}{r}0,82 \\
0,79 \\
0,79 \\
0,72 \\
0,70 \\
0,68 \\
0,63 \\
0,63 \\
0,58 \\
0,56 \\
0,56 \\
0,50\end{array}$ \\
\hline \multicolumn{2}{|c|}{ Varianza explicada $=35,94 \%$} & & inza explicada $=21,4 \%$ \\
\hline
\end{tabular}


decidió mantener esta estructura, cuyos aspectos más relevantes se comentan a continuación.

En la Tabla 1 se muestra la estructura y saturación del INPIAS-Parte 1. La mayor parte de los items que recoge el primer factor hacen referencia a intrusiones de tipo verbal (pensamientos), que se activan en presencia de indicios relacionados con la comida (durante o después de comer), verse el propio cuerpo, o al hallarse en presencia de terceros (lo que suscita un proceso de comparación). Muchos de los pensamientos adquieren la forma de imperativos o desiderata («tengo que...»» «no debería...»), y de duda («iestaré...?). Finalmente, sólo 5 de las 38 intrusiones adquieren formato de impulso a actuar: 3 sobre hacer ejercicio, 1 sobre hacer dieta, y 1 sobre ocultar parte del propio cuerpo. Podríamos considerar el contenido de factor como un conjunto de intrusiones que tienen que ver con el tipo de creencias, valores y comportamientos relacionados con el ya acuñado término de «descontento normativo», es decir, una serie de creencias y actitudes que son producto de la actual presión social por mantenerse en un cuerpo delgado y que afecta al gran conjunto de la población. Es por este motivo que denominamos al Factor 1 del INPIAS-Parte 1, Intrusiones de descontento normativo.

Sin embargo, el segundo factor recoge básicamente impulsos ( 8 items de los 12), cuyos contenidos hacen referencia a comportamientos que pueden calificarse como patológicos: conductas purgativas-restrictivas (ayunar, vomitar, laxarse), de acumulación e ingesta excesiva, o de recuento de calorías ante la comida. Por lo que refiere a las intrusiones en forma de pensamiento, sus contenidos refieren asimismo a conductas purgativas y restrictivas (vomitar, auto-instrucción de no comer), y un último pensamiento es francamente auto-despreciativo («mi aspecto da asco»). A este factor lo hemos denominado Impulsos de psicopatología alimentaria, ya que parece estar haciendo referencia a intrusiones cuyos contenidos se asemejan bastante a los que experimentan las personas con un TA.

- INPIAS-Parte 2-A. Consecuencia emocional, interferencia, y valoraciones disfuncionales de la intrusión más molesta.
También en este caso la estructura bi-factorial resultó ser la más adecuada (véase Tabla 2). Ambos factores correlacionaron estrechamente $(r=0,53)$. Por esta razón, se decidió mantener la rotación promax como la más adecuada.

En el primer factor se agruparon todas las valoraciones de tipo emocional asociadas con el PIA más molesto, además de las relacionadas con el significado personal o auto-revelador del mismo (ítems 9, 16, 11 y 14), y los items relacionados con el esfuerzo para mantener bajo control dicha intrusión (interferencia). A este factor lo hemos denominado Carga emocional de la intrusión y su significado personal. El segundo factor agrupó las cuatro valoraciones más claramente disfuncionales sobre la aparición del PIA (amenaza, fusión pensamiento-acción, y responsabilidad para impedir consecuencias negativas asociadas a la intrusión). A este factor lo hemos denominado Profecía cumplida y responsabilidad personal.

Tabla 2. Análisis factorial exploratorio (pesos factoriales),

INPIAS-Parte 2-A: Consecuencias producidas por el PIA más molesto $(N=358)$

\begin{tabular}{rcc}
\hline Parte 2-A: Consecuencias emocionales y valoraciones \\
\hline Ítem & Factor 1 & Factor 2 \\
\hline 1 & 0,85 & \\
2 & 0,82 & \\
3 & 0,80 & \\
9 & 0,78 & \\
16 & 0,76 & \\
8 & 0,68 & \\
6 & 0,68 & \\
4 & 0,66 & \\
5 & 0,65 & \\
14 & 0,59 & \\
11 & 0,57 & \\
7 & $-9,55$ & 0,87 \\
12 & & 0,87 \\
15 & & 0,59 \\
10 & & 0,57 \\
13 & & \\
\hline
\end{tabular}

Varianza explicada $=40 \%$ Varianza explicada $=21,1 \%$ Varianza explicada total $=61,14 \%$

- INPIAS-Parte 2-B. Estrategias para controlar la intrusión más molesta

La estructura más interpretable (promax) fue la de cuatro factores. El primer factor mostró 
correlaciones pequeñas con el resto $(r$ entre 0,15 y 0,30$)$, mientras que no se detectaron correlaciones entre los otros tres factores. En consecuencia, se decidió aplicar una rotación varimax, que presentó una estructura prácticamente idéntica a la preliminar y con igual porcentaje de varianza explicada $(58,24 \%)$. Según esta estructura (véase Tabla 3), el primero de los componentes se destaca claramente del resto por su importancia en cuanto al porcentaje de varianza explicada, mientras que el cuarto agrupó únicamente dos items, explicando tan sólo un 6,54 $\%$ de la varianza. No obstante, se decidió mantener esta estructura dado que los sucesivos ensayos con otra modalidad de rotación (equamax) y ampliando o reduciendo el número de factores a extraer, seguían mostrando una estructura prácticamente idéntica en la que los dos items del cuarto factor permanecían juntos y separados del resto («hago lo que me dicta la intrusión», $\mathrm{y}$ «no hago nada para librarme de ella»). Por otro lado, dado que había dos items con un contenido prácticamente idéntico, y como era de esperar, ambos aparecían en el mismo factor, se decidió eliminar el de menor saturación.
Por lo que se refiere al contenido de los factores, el primero de ellos agrupa estrategias de control que suelen calificarse como normalizadoras y/o adaptativas ante un evento cognitivo molesto (re-evaluar, auto-tranquilizarse, relajarse, preguntar a otros, etc.), si bien incluye también auto-castigo leve («me riño»), evitación y centrarse en otras preocupaciones. A este factor lo hemos denominado, por tanto Estrategias de control normalizadoras. El Factor 2 agrupó estrategias algo más problemáticas dirigidas a intentar mantener bajo control la intrusión molesta (ocultar, suprimir, compulsión encubierta) y que claramente requieren y consumen mucho esfuerzo y recursos mentales, junto a otra más adaptativa (distracción). A este factor lo hemos denominado Estrategias de neutralización y supresión. El Factor 3 agrupa los comportamientos que, en un contexto obsesivo, entrarían en la consideración de rituales compulsivos (ordenar, limpiar, repetir, comprobar). Por ello su denominación es la de Rituales no alimentarios. Finalmente, el Factor 4 indica claramente una especie de rendición ante el contenido de la intrusión, es decir, hacer lo que «dicta» la intrusión, probablemente alguna

Tabla 3. Análisis factorial exploratorio (saturaciones), INPIAS-Parte B: Estrategias ante el PIA más molesto $(N=358)$

\begin{tabular}{rcccc}
\hline \multicolumn{5}{c}{ Parte 2-B: Consecuencias emocionales y valoraciones } \\
\hline Item & Factor 1 & Factor 2 & Factor 3 \\
\hline 5 & 0,75 & & & \\
8 & 0,73 & & & \\
7 & 0,72 & 0,49 & & \\
6 & 0,67 & 0,58 & & \\
9 & & & & \\
16 & 0,56 & & & \\
11 & 0,54 & & & \\
13 & 0,52 & 0,71 & & \\
12 & 0,48 & 0,68 & & \\
15 & & 0,62 & & \\
1 & & 0,59 & 0,82 & \\
2 & & 0,55 & 0,78 & 0,62 \\
17 & & & 0,72 & \\
14 & & & & \\
3 & & & & \\
$4 \mathrm{a}$ & & & & \\
$4 \mathrm{~d}$ & & & & \\
$4 \mathrm{~b}$ & & & & \\
$4 \mathrm{c}$ & & & & \\
10 & & & & \\
\hline
\end{tabular}

Varianza explicada $=22,38 \%$ Varianza explicada $=16,02 \%$ Varianza explicada $=13,30 \%$ Varianza explicada $=6,54 \%$

Varianza explicada total $=58,24 \%$ 
cosa que anule su anuncio y presencia. A este factor lo denominamos No controlar; obedecer a la intrusión.

\section{Fiabilidad: consistencia interna y estabilidad temporal}

Los coeficientes de los dos factores del INPIAS-Parte 1 fueron 0,98 y 0,92; de los dos factores del INPIAS-Parte 2-A 0,88 y 0,77; y de los cuatro del INPIAS-Parte 2-B fueron, en su orden, $0,84,0,86,0,80$ y 0,40 , respectivamente.

Por lo que respecta a su estabilidad temporal (dos semanas) se obtuvieron los siguientes CCI: en los dos factores del INPIAS-Parte 1: 0,91 y 0,80 ; en los dos factores del INPIASParte 2-A: 0,93 y 0,91; y en los cuatro factores del INPIAS-Parte 2-B: 0,84, 0,83, 0,90, 0,81 . Por tanto, hay que destacar que, aunque el Factor 4 de la Parte 2-B (dos items e inver- samente relacionados) presenta una consistencia muy baja, sin embargo la repetibilidad o estabilidad temporal del cuestionario ha mostrado ser excelente, incluyendo el mencionado Factor 4, lo que indica que la medida no se ve afectada por el paso del tiempo.

Validez convergente y discriminante. Relación con medidas de TA y TOC

Todas las asociaciones de la Parte 1 del INPIAS (contenido de intrusiones y frecuencia) con las medidas de patología alimentaria (RS, EAT y MBSRQ) y obsesivo-compulsiva (CBO$\mathrm{CI})$ resultaron significativas. No obstante, atendiendo al tamaño de los coeficientes, las correlaciones fueron en general mayores con los instrumentos de TA (y muy especialmente con los factores relacionados con seguir una dieta) que con los de TOC (véase Tabla 4).

Tabla 4. Correlaciones entre INPIAS-Parte 1 (frecuencia intrusiones)

y medidas de trastornos alimentario y obsesivo-compulsivo

\begin{tabular}{lcc}
\hline MEDIDAS DE TA & $\begin{array}{c}\text { Factor 1 } \\
\text { Descontento } \\
\text { normativo }\end{array}$ & $\begin{array}{c}\text { Factor } 2 \\
\text { Impulsos } \\
\text { Psicopatología alimentaria }\end{array}$ \\
\hline EAT Factor I: Dieta & $0,64^{* * *}$ & $0,41^{* * *}$ \\
EAT Factor II: Bulimia y preocupación por el alimento & $0,51^{* * *}$ & $0,39^{* * *}$ \\
EAT Factor III: Control oral & $0,24^{* * *}$ & $0,23^{* * *}$ \\
RS (Implicación en la dieta) & $0,62^{* * *}$ & $0,46^{* * *}$ \\
RS (Fluctuación en el peso) & $0,47^{* * *}$ & $0,32^{* * *}$ \\
MBSRQ. Evaluación de la apariencia & $-0,49^{* * *}$ & $-0,31^{* * *}$ \\
MBSRQ. Orientación a la apariencia & $0,28^{* * *}$ & $0,26^{* * *}$ \\
MBSRQ. Satisfacción áreas corporales & $-0,50^{* * *}$ & $-0,32^{* * *}$ \\
MBSRQ Preocupación por sobrepeso & $0,63^{* * *}$ & $0,41^{* * *}$ \\
MBSRQ. Auto-clasificación del peso & $0,35^{* * *}$ & $0,18^{* *}$ \\
\hline
\end{tabular}

MEDIDAS DE TOC

CBOCI-. Obsesiones

CBOCI-. Compulsiones

$\begin{array}{ll}0,35 * * * & 0,32 * * * \\ 0,29 * * * & 0,27 * * *\end{array}$

Nota: $* \mathrm{p}<0,02 ; * * \mathrm{p}<0,001 ; * * * \mathrm{p}<0,0001$. EAT: Eating Attitudes Test; RS: Restraint Scale; MBSRQ: Multidimensional Body-Self Relations Questionnaire; C-BOCI: Clark-Beck Obsessive Compulsive Inventory.

En cuanto a las consecuencias emocionales y valoraciones disfuncionales del PIA más molesto (INPIAS-Parte 2-A), los dos factores de esta parte correlacionaron significativamente con todas las dimensiones de creencias disfun- cionales obsesivas (ICO), si bien la mayor parte de los coeficientes fue de tamaño pequeño (véase Tabla 5). Los coeficientes mayores se obtuvieron con las creencias de Sobre-estimación de la amenaza, seguidas de las creencias 
sobre la Importancia de controlar la intrusión más desagradable. También fueron significativas, aunque moderadas, todas las correlaciones con las subescalas de la medida de perfeccionismo (MPS), siendo de tamaño entre pequeño y medio la mayor parte de los coeficientes. Las asociaciones más importantes fueron las obtenidas con las subescalas de Preo- cupación por los errores y Dudas sobre las propias acciones.

Por lo que se refiere al examen de las asociaciones con las estrategias de control ante el PIA más desagradable (INPIAS-Parte 2-B), únicamente se calcularon para las medidas relacionadas con TA (véase Tabla 6). Las subescalas del EAT correlacionaron significativamente

Tabla 5. Correlaciones entre INPIAS-Parte 2-A

(Consecuencias y valoraciones del PIA más molesto) y medidas de creencias y perfeccionismo

\begin{tabular}{lcc}
\hline & $\begin{array}{c}\text { Factor } 1 \\
\text { Carga emocional } \\
\text { significado personal }\end{array}$ & $\begin{array}{c}\text { Factor } 2 \\
\text { Profecía cumplida }\end{array}$ \\
y responsabilidad personal
\end{tabular}

Nota: $* \mathrm{p}<0,02 ; * * \mathrm{p}<0,001 ; * * * \mathrm{p}<0,0001$. ICO: Inventario de Creencias Obsesivas; MPS: Multidimensional Perfectionism Scale.

Tabla 6. Correlaciones entre INPIAS-Parte 2-B (Estrategias ante el PIA más molesto) y medidas TA

\begin{tabular}{|c|c|c|c|c|}
\hline$E A T$ & $\begin{array}{c}\text { Factor } 1 \\
\text { Normalizadoras }\end{array}$ & $\begin{array}{c}\text { Factor } 2 \\
\text { Neutralización/ } \\
\text { Supresión }\end{array}$ & $\begin{array}{c}\text { Factor } 3 \\
\text { Rituales } \\
\text { No alimenta. }\end{array}$ & $\begin{array}{c}\text { Factor } 4 \\
\text { No controlar, } \\
\text { dejarse llevar }\end{array}$ \\
\hline Hacer Dieta & $0,16^{*}$ & $0,28 * * *$ & $0,23 * *$ & $0,19 * *$ \\
\hline Bulimia & $0,16^{*}$ & $0,23 * * *$ & $0,187^{* *}$ & $0,15^{* *}$ \\
\hline Control Oral & 0,02 & 0,06 & $-0,007$ & $0,14^{*}$ \\
\hline Punt. total & $0,15^{*}$ & $0,26^{* * *}$ & $0,20 * *$ & $0,20 * *$ \\
\hline \multicolumn{5}{|l|}{ MBSRQ } \\
\hline Evaluac. Apariencia & $-0,12^{*}$ & $-0,19 * *$ & $-0,18 * *$ & $-0,61$ \\
\hline Orientac. Apariencia & $0,15^{*}$ & $0,20 * *$ & $0,14 * *$ & $0,18 * *$ \\
\hline Satisfac. áreas corporales & $-0,15^{*}$ & $-0,21 * *$ & $-0,22 * *$ & $-0,11^{*}$ \\
\hline Preocupación sobrepeso & $0,19 * *$ & $0,23 * * *$ & $0,27 * * *$ & $0,20 * *$ \\
\hline Autoclasificación del peso & 0,07 & $0,18 * *$ & $0,16^{*}$ & 0,008 \\
\hline
\end{tabular}

Nota: $* \mathrm{p}<0,02 ; * * \mathrm{p}<0,001 ; * * * \mathrm{p}<0,0001$. EAT: Eating Attitudes Test; MBSRQ: Multidimensional Body-Self Relations Questionnaire. 
con todas las estrategias de control, a excepción de la escala de Control oral que tan sólo se asoció de manera significativa con el Factor 4 (No controlar, dejarse llevar) del INPIAS. En cuanto al MBSRQ, las subescalas en las que se valora de forma positiva el propio cuerpo (Evaluación de la apariencia y Satisfacción áreas corporales) correlacionaron negativamente con las estrategias de control del PIA más molesto. El resto de escalas correlacionaron positiva y significativamente con las estrategias del INPIAS, a excepción de la escala de Autoclasificación del peso, que tan sólo mantuvo relación significativa con las estrategias de Neutralización/supresión y Rituales no alimentarios del INPIAS.

Por último, en la Tabla 7 se muestran las correlaciones entre el INPIAS y otras variables de interés. De la misma manera que en los anteriores análisis, cuando las correlaciones fueron significativas, oscilaron entre moderadas a bajas, como en el caso de la preocupación ansiosa (worry). Este último dato es de interés en tanto que indica que las intrusiones que recogen el cuestionario INPIAS se evalúan como tales y no como preocupaciones ansiosas, siendo por tanto distintos fenómenos. Por otra parte, mientras que la variable del peso (IMC) mantuvo una relación significativa y positiva con «Descontento normativo», «Impulsos de psicopatología alimentaria», y «Carga emocional y significado personal», la variable edad correlacionó negativamente con estos mismos factores. Con el resto de factores del INPIAS, las relaciones con edad e IMC no fueron significativas.

\section{Diferencias en el INPIAS entre personas con alta restricción (población subclínica) y con baja restricción (población sin riesgo)}

Los participantes en el estudio se dividieron en dos grupos sobre la base de la puntuación en la RS, que como ya se comentó, se utiliza como instrumento de screening para detectar población de riesgo o subclínica con respecto a la psicopatología de los TA. De esta manera, de los 358 participantes, 55 puntuaron por encima del criterio (grupo subclínico), y 303 por debajo (población sin riesgo, SR). El grupo subclínico lo componían 7 hombres $(12,7 \%)$ y 48 mujeres $(87,3 \%)$, con una media de edad de 23,33 $(D T=3,68$; rango $15-35)$, y una media de IMC de $23,52(D T=3,47$; rango 17,58-32,11). La mayoría $(85,5 \%)$ estaba soltera o sin pareja, tenían estudios superiores $(58,2 \%)$, y un nivel económico medio (69,1\%). El grupo SR lo componían 84 hombres $(28,1 \%)$ y 215 mujeres $(71,9 \%)$, con una edad de $24(D T=4,1$; rango 14-38), y un IMC de 21,92 ( $D T=2,84$; rango $17,03-32,87)$. La mayoría estaba soltera o sin pareja $(85,3 \%)$, tenían estudios superiores $(65,6 \%)$, y su nivel económico era medio $(72,2 \%)$. No se constataron diferencias entre los dos grupos en las variables socio-demográficas, aunque sí en el IMC, que fue mayor en el grupo subclínico $(23,52 ; D T=3,47$ vs. 21,92 ; $\left.D T=2,84) ; t_{68,06}=-3,21 ; \mathrm{p}<0,002\right)$.

Antes de proceder a analizar las diferencias entre los grupos SR y subclínico, se examinaron las posibles diferencias dentro del grupo más amplio ( $N$ : 303; grupo SR) en las principales

Tabla 7. Correlaciones entre INPIAS y Edad, IMC, Auto-estima y «Preocupación ansiosa»

\begin{tabular}{|c|c|c|c|}
\hline INPIAS & Edad & $I M C$ & $P S W Q$ \\
\hline Parte 1. F 1: Intrusiones & $-0,12^{*}$ & $0,23 * * *$ & $0,26 * * *$ \\
\hline \multicolumn{4}{|l|}{ Descontento normativo. } \\
\hline Parte 1: F 2: Impulsos de Psicopatología alimentaria & $-0,16^{* *}$ & $0,13 * *$ & $0,20 * * *$ \\
\hline Parte 2-A. F 1: Carga emocional y sigdo. personal & $-0,15^{* *}$ & $0,13 * *$ & $0,35 * * *$ \\
\hline Parte 2-A. F 2: Profecía cumplida y responsabilidad & $-0,07$ & 0,01 & $0,30 * * *$ \\
\hline Parte 2-B. F 1: Estrategias normalizadoras & $-0,02$ & 0,008 & $0,31 * * *$ \\
\hline Parte 2-B. F 2: Estrategias Neutralización/supresión & $-0,04$ & 0,07 & $0,30 * * *$ \\
\hline Parte 2-B. F 3: Rituales no alimentarios & 0,08 & 0,09 & $0,17 * *$ \\
\hline Parte 2-B. F 4: No controlar, obedecer & $-0,04$ & $-0,09$ & 0,09 \\
\hline
\end{tabular}

Nota: ${ }^{*} \mathrm{p}<0,02 ;{ }^{*} \mathrm{p}<0,001 ; * * * \mathrm{p}<0,0001$. IMC: Índice de Masa Corporal; PSWQ: Penn State Worry Questionnaire. 
variables demográficas. Este análisis preliminar se realizó con el objetivo de controlar el peso que las posibles diferencias detectadas pudieran tener sobre las diferencias entre la población sin riesgo y el grupo subclínico en el INPIAS. Para el cálculo de frecuencia media de los pensamientos intrusos se dividió la suma de las frecuencias partido por el número de pensamientos que habían sido experimentados con una frecuencia diferente de 0 (nunca).

No se encontraron diferencias en función de la edad, nivel de educación, nivel económico, o estado civil, pero sí en cuanto al sexo en algunas variables. Así, aunque las mujeres puntuaron más en todas las escalas, sólo se encontraron diferencias estadísticamente significativas en la Carga emocional de las intrusiones y su significado personal (mujeres: 0,$98 ; D T=0,54$; hombres: 0,$79 ; D T=0,48 ; t_{300}=2,88 ; \mathrm{p}<0,01$ ) $\mathrm{y}$ en dos de los cuatro factores de las estrategias de afrontamiento: Neutralización y supresión (mujeres: 1,$19 ; D T=0,89$; hombres: 0,$\left.95 ; D T=0,82 ; t_{300}=2,12 ; \mathrm{p}<0,01\right)$ y Rituales no alimentarios (mujeres: 0,$41 ; D T=0,65$; hombres: 0,$\left.22 ; D T=0,42 ; t_{321,800}=2,94 ; \mathrm{p}<0,01\right)$. No existieron diferencias, por tanto, ni en la frecuencia y tipo de intrusiones, ni en el resto de valoraciones y estrategias, que son utilizadas por igual por hombres y por mujeres.

A continuación se expone la frecuencia de intrusiones de carácter alimentario en los dos grupos objeto de análisis, SR y subclínicos. Por lo que se refiere los PIA más frecuentes y los más molestos, los que más frecuentemente fueron experimentados por el grupo SR resultaron ser los relacionados con pensamientos sobre la necesidad de hacer ejercicio, ya sea cuando se está comiendo (ítem 9; frecuencia media $=2,25$ ) o frente al espejo (ítem 30; frecuencia media $=$ $2,15)$. Los menos frecuentes fueron dos ítems relacionados con impulsos de vomitar (ítem 22; frecuencia media $=0,06$ ) o tomar un laxante después de haber comido (ítem 23; frecuencia media $=0,08)$. En consecuencia, estas intrusiones, que suelen ser características de patología alimentaria, no son experimentadas prácticamente nunca por la población SR. En cuanto al grupo subclínico, las frecuencias medias fueron, en general, mayores. La experimentada con más frecuencia fue de nuevo el ítem 9 («Tengo que hacer ejercicio», frecuencia media $=4,02$ ), seguida del 19 («Tengo que ponerme a dieta», frecuencia media: 4) y el 46 («Sería maravilloso estar delgado»; frecuencia media $=3,91)$. Las menos frecuentes fueron las recogidas en los ítems 12 (Almacenar comida; frecuencia media $=0,64)$ y 23 (Laxarse; frecuencia media $=0,69)$. Desde el punto de vista de la puntuación total en los dos factores de la Parte 1, ambos grupos presentaron diferencias significativas en cuanto a la frecuencia de cada tipo de PIA.

Por lo que se refiere al PIA escogido como el más perturbador o molesto, el grupo SR escogió dos relacionados con el ejercicio, que además estaban entre los más frecuentemente experimentados (ítems 9 y 30). En el grupo subclínico, hubo una mayor variabilidad en cuanto al contenido del PIA escogido como el más molesto, pero destacan las intrusiones que afirman el hecho de que se está «gordo» suscitada al ver el propio cuerpo, y con la necesidad de hacer dieta (ítems 19, 44, y 46), que a su vez fueron las intrusiones más frecuentes en este grupo.

En cuanto al análisis de las consecuencias ante la intrusión más molesta, el grupo SR presentó bajas puntuaciones en las consecuencias emocionales negativas y las valoraciones disfuncionales ante su PIA más molesto. El rango de puntuaciones media osciló entre 0,17 y 1,23 y la mayor puntuación se observó en el éxito al controlar la intrusión $(M=2,53)$. El grupo subclínico obtuvo mayores puntuaciones en todas estas variables (rango entre 0,65 y 2,47 ), destacando la mayor reactividad emocional, tanto en intensidad como en variedad, así como el significado personal otorgado a la intrusión $(M=2,05)$.

La estrategia de control más habitual en el grupo SR fue la de razonar sobre la intrusión $(M=1,56)$, mientras que estas personas no suelen realizar «rituales no alimentarios. En el grupo subclínico, las estrategias menos utilizadas fueron las de Rituales no alimentarios, y la que utilizaron con más frecuencia fue la Distracción conductual $(M=2,13)$.

Por último se llevó una prueba $t$ para analizar los resultados obtenidos en todas las subescalas del INPIAS en los grupos SR y subclínico (véase Tabla 8). El grupo subclínico obtuvo puntuaciones significativamente más altas que el 
Tabla 8. Descriptivos y diferencias entre población sin riesgo y subclínicos en el INPIAS

\begin{tabular}{|c|c|c|c|c|c|c|c|c|}
\hline & \multicolumn{2}{|c|}{$\begin{array}{c}\text { INPIAS-Parte 1: } \\
\text { Frecuencia Intrusiones }\end{array}$} & \multicolumn{2}{|c|}{$\begin{array}{c}\text { INPIAS-Parte 2: } \\
\text { Consecuencias y valoraciones }\end{array}$} & \multicolumn{2}{|c|}{$\begin{array}{l}\text { INPIAS-Parte 3: } \\
\text { Estrategias }\end{array}$} & \multirow[b]{2}{*}{$\begin{array}{c}\text { Rituales } \\
\text { no alimentario }\end{array}$} & \multirow[b]{2}{*}{$\begin{array}{l}\text { No controlar: } \\
\text { obedecer }\end{array}$} \\
\hline & $\begin{array}{l}\text { Descontento } \\
\text { Normativo }\end{array}$ & $\begin{array}{c}\text { Impulsos } \\
\text { Psicopatología } \\
\text { Alimentaria }\end{array}$ & $\begin{array}{c}\text { Carga } \\
\text { emocional y } \\
\text { Sgdo. Personal }\end{array}$ & $\begin{array}{c}\text { Profecía } \\
\text { cumplida } \\
\text { y responsab. }\end{array}$ & $\begin{array}{c}\text { Estregias } \\
\text { Control } \\
\text { Normaliza }\end{array}$ & $\begin{array}{l}\text { Neutraliza } \\
\text { Supresión }\end{array}$ & & \\
\hline $\begin{array}{l}\text { SR } \\
(N=303)\end{array}$ & $1,88(0,66)$ & $1,10(0,96)$ & $0,93(0,53)$ & $0,34(0,47)$ & $1,00(0,76)$ & $1,13(0,88)$ & $0,36(0,60)$ & $1,71(0,69)$ \\
\hline $\begin{array}{l}\text { Subclínicos } \\
(N=55)\end{array}$ & $3,30(0,90)$ & $2,18(1,25)$ & $1,96(0,64)$ & $0,93(0,87)$ & $1,45(0,72)$ & $1,87(0,76)$ & $0,82(0,79)$ & $2,10(0,67)$ \\
\hline${ }^{t}(g l)$ & $\begin{array}{c}11,16 \\
(65,129)\end{array}$ & $\begin{array}{l}7,25 \\
(356)\end{array}$ & $\begin{array}{l}12,59 \\
(355)\end{array}$ & $\begin{array}{c}4,83 \\
(59,93)\end{array}$ & $\begin{array}{l}4,06 \\
(355)\end{array}$ & $\begin{array}{c}5,82 \\
(355)\end{array}$ & $\begin{array}{c}4,09 \\
(65,96)\end{array}$ & $\begin{array}{l}3,81 \\
(355)\end{array}$ \\
\hline
\end{tabular}

Nota: Los datos de las dos primeras filas se expresan como Media $(D T)(\mathrm{p}<0,0001$ para todas las variables $) . \mathrm{SR}=\sin$ riesgo.

grupo SR en todas las escalas del INPIAS. Se llevaron a cabo ANCOVAS controlados por edad, IMC y obsesividad (medido con el CBOCI), y las diferencias antes comentadas siguieron resultando significativas y, por tanto, confirmadas.

\section{DISCUSIÓN}

El objetivo fundamental de este trabajo ha sido desarrollar y validar un instrumento de autoinforme para detectar la presencia, en la población general, de intrusiones con contenido análogo a las que experimentan las personas con trastornos alimentarios (PIAs), así como sus repercusiones emocionales y las estrategias de control y/o neutralización que promueven dichas intrusiones. El instrumento que hemos desarrollado, y que hemos denominado como Inventario de Pensamientos Intrusos Alimentarios (INPIAS) ha mostrado tener unas cualidades psicométricas básicas (consistencia interna y repetibilidad) entre buenas y excelentes, lo que nos permite estar razonablemente seguras de su adecuación como instrumento de medida.

Los contenidos de las intrusiones alimentarias examinadas se agruparon en dos conjuntos claramente diferenciados: por un lado, las que se refieren a deseos y/o comportamientos normativos y socialmente deseables (hacer ejercicio, dieta, comer adecuadamente, etc.) con un formato preferentemente verbal (i.e., pensamientos), y a las que hemos denominado como PIAs de Descontento normativo, y por otro, un conjunto de intrusiones relacionados con conductas compensatorias y purgativas, con un formato básicamente de impulso a actuar y que hemos catalogado como Impulsos de psicopatología alimentaria, ya que se encuentran mucho más cercanas que las primeras a lo que dicen experimentar las personas con TA. Por su parte, las consecuencias emocionales y las valoraciones disfuncionales que tienen y/o hacen las personas en respuesta a la intrusión que escogieron como más desagradable o perturbadora (Parte 2-A del INPIAS) se agruparon asimismo en dos conjuntos o factores: el primero englobó las emociones negativas, la interferencia, y la atribución de un significado personal al contenido de dicha intrusión, mientras que el segundo asoció las valoraciones disfuncionales que, por decirlo gráficamente, «anuncian» los efectos negativos del contenido de la intrusión con la responsabilidad personal que conlleva el tenerlas y mantenerlas bajo control. Por último, las estrategias de control y/o neutralización que las personas ponen en marcha ante la intrusión más molesta se agruparon en cuatro conjuntos: estrategias adaptativas ante un evento cognitivo molesto; neutralización y supresión activas; las que se centran en rituales típicos del espectro compulsivo; y finalmente no hacer nada, o hacer lo que dicta la intrusión.

Con respecto a la validez convergente y discriminante, el INPIAS ha mostrado unas relaciones significativas, pero moderadas, con otros 
instrumentos pertinentes tanto para la evaluación de los TA como del TOC, y en especial para los primeros. Ello significa que aunque el nuevo instrumento está evaluando aspectos relacionadas con ambos conjuntos de instrumentos, la moderación de los coeficientes indica también que el INPIAS recoge y valora características que no se contemplan adecuadamente en los instrumentos más habituales de los TA y del TOC. En concreto, en cuanto a la parte del INPIAS que evalúa el tipo y frecuencia de PIAs, las correlaciones siempre han sido algo más elevadas con los instrumentos de TA que con los de TOC, en especial por lo que se refiere a las intrusiones de descontento normativo, mientras que en el caso de los impulsos de psicopatología alimentaria, la magnitud de correlaciones está más equilibrada en cuanto a instrumentos de TA y TOC. Por su parte, las relaciones de la parte del INPIAS sobre consecuencias y valoraciones con las creencias típicamente obsesivas y el perfeccionismo, se observa que tanto la Carga emocional y significado personal como la Profecía cumplida y responsabilidad personal que se producen a consecuencia de la experimentación del PIA más molesto, presentan correlaciones entre bajas y moderadas, pero significativas, destacándose la mayor asociación con las valoraciones obsesivas de la importancia de controlar los pensamientos, el sobrestimar la amenaza, y con las dimensiones de Dudas sobre acciones y Preocupación por los errores del perfeccionismo (Shafran, 2002; Shafran, Bryant-Waugh, Lask, y Arscott, 1995).

También ha resultado de interés las relaciones encontradas entre las estrategias de control del PIA más molesto y los instrumentos de evaluación del ámbito de los TA. Hay que destacar que, a diferencia de los que sucede en el resto de escalas del EAT, la de Control oral sólo mantiene una relación significativa con la estrategia No controlar, obedecer del INPIAS. Mientras que la subescala Hacer dieta del EAT refleja la necesidad de restringir la ingesta porque la persona se siente gorda, y la de Bulimia expresa la preocupación por la comida y la falta de control sobre el impulso de vomitar, atracarse, etc., la escala de Control oral evalúa la sensación de control que tiene la persona sobre la comida, además de la percepción de que los demás estén pendientes de la persona para que coma. Es decir, lo que indica esta asociación entre el control sobre la comida y el obedecer a la intrusión es que la persona considera positiva, esta intrusión y por tanto no tiene necesidad de controlarla o neutralizarla. Esto revela, por una parte, la ya postulada funcionalidad del trastorno (el tener bajo control algún aspecto en la vida) (Slade, 1982) y, por otra, la naturaleza egosintónica de estos contenidos ideativos que los diferenciaría de los típicamente obsesivos que son, mayoritariamente egodistónicos (Belloch et al., 2004; Belloch, Morillo y García Soriano 2007). También es de destacar el análisis de la imagen corporal que surge a través del MBSRQ, cuyas escalas correlacionan significativamente con las estrategias de control del INPIAS, a excepción de la Autoclasificación del peso que tan sólo se muestra significativa con respecto a las estrategias de Neutralización y supresión y la de Rituales no alimentarios, es decir con las dos más patológicas. Teniendo en cuenta que la autoclasificación del peso se basa en creo que mi peso es..., es decir en la creencia de lo que la persona considera pesar, estos datos apoyan la relevancia que tienen la implicación de la persona en conductas de dieta (sobre todo la «necesidad» de seguirla para alcanzar un cuerpo delgado o huir de un cuerpo que siempre se considera demasiado gordo) en la gestación de un TA (Herman y Polivy, 2007; Vartaniana, Herman y Polivy, 2007).

Finalmente, ha resultado especialmente interesante el análisis de las relaciones con la edad y el IMC. La edad, más concretamente tener más años, es como se ha mostrado en otros trabajos (Benedito, Perpiñá, Botella, y Baños, 2003; Sirton y Birch, 2005) un factor protector ante la experimentación de insatisfacción corporal, la implicación en conductas de riesgo y, como vemos aquí, a experimentar intrusiones alimentarias. Por otra parte, el exceso de peso es una variable que favorece la presencia y frecuencia de intrusiones, lo cual es lógico en tanto que el exceso de peso, y el temido «michelín» es una condición física estigmatizada socialmente (Tanofsky-Kraff, Yanovski, Wil- 
fley, Marmarosh, Morgan, y Yanovski, 2004; Joquera, Baños, Perpiñá y Botella, 2005). Ahora bien, ni la edad ni el peso tienen relación alguna con las estrategias con las que las personas intentan combatir su PIA más molesto, ni con la valoración disfuncional que anticipa los peligros que la persona asocia a la intrusión, lo cual indica un nivel cualitativamente distinto en la escalada de este continuo, ya que la psicopatología de los TA, como sabemos, se puede producir en cualquier edad y en cualquier rango de peso. Finalmente destacar la baja/ moderada asociación, pero significativa (con la excepción de la estrategia de ceder a la intrusión) con la medida de la preocupación ansiosa (worry) reflejando, por tanto, que lo que está evaluando el INPIAS son productos cognitivos diferentes a las «preocupaciones ansiosas» de contenido alimentario.

Por otro lado, es interesante resaltar que la única variable sociodemográfica en la que se detectaron diferencias en la población general fue en la referida al sexo, ya que las mujeres puntuaron significativamente más en la carga emocional de las intrusiones y su significado personal y en dos estrategias de control: Neutralización y supresión; y Rituales no alimentarios. Estos datos muestran una vez más que, aunque hombres y mujeres compartimos los estereotipos socio-culturales con respecto a un cuerpo bello y la importancia de «cuidarse» en la alimentación y en el ejercicio, son las mujeres las que más significado personal otorgan a estos aspectos, que a su vez tienden a tener mayores repercusiones en las reacciones emocionales adversas (McKinley, 2002). Por otra parte, despuntan dos estrategias que, según nuestros datos, son más típicas de mujeres: las de los rituales de ordenar, limpiar, comprobar, y estrategias que requieren esfuerzo y concentración para deshacerse de la intrusión.

Un objetivo adicional del estudio fue examinar si había diferencias entre personas en riesgo de padecer un TA (población subclínica) y personas sin riesgo, en los diversos aspectos que evalúa el INPIAS: frecuencia de PIAs, y consecuencias emocionales y comportamentales (estrategias de control). Los resultados obtenidos indican que el cuestionario es capaz de di- ferenciar adecuadamente entre la población con y sin riesgo de padecer un TA. Pero además, las elevadas puntuaciones obtenidas en la Parte 1 del INPIAS por el grupo de personas catalogadas como subclínicas, indican que la experimentación frecuente de intrusiones alimentarias podría estar en la base de una elevada vulnerabilidad a padecer un TA, apoyando con ello no solo el enfoque cognitivo de estos trastornos, sino también la comunalidad existente entre los TA y el TOC (Jones et al., 2005; Speranza et al., 2001).

El perfil de los PIAs en cada grupo indica que los sujetos sin riesgo de presentar un TA experimentaba intrusiones sobre hacer ejercicio al comer, o al verse, con una frecuencia mínima (algunas veces al año), y por contra no han experimentado nunca intrusiones relacionadas con los impulsos de conductas purgativas. De hecho, sus PIAs valorados como más molestos tienen que ver con el mero hecho de pensar en hacer ejercicio físico, y estas intrusiones provocan una baja tasa de emociones negativas (por ej., nunca han experimentado vergüenza por pensar en ello), poseen escaso significado personal (en el sentido de que no forman parte de su auto-concepto), y generan poca credibilidad sobre las consecuencias adversas en el futuro a causa de la intrusión. Su máxima puntuación se da precisamente en el éxito que tienen en controlar el PIA, es decir, que son perfectamente capaces de «olvidarse» de hacer ejercicio. En cuanto a las estrategias, la más frecuentemente utilizada es la de razonar la intrusión, es decir, la que se ha mostrado como claramente adaptativa e indicadora de mejoría o normalización (Belloch et al., 2007; Purdon y Clark, 2001).

Por lo que respecta al grupo subclínico, también presentó intrusiones con respecto al ejercicio, aunque con una frecuencia mucho mayor que los SR, además de intrusiones sobre hacer dieta y sobre «lo magnífico» que es estar delgado, habiendo ya experimentado incluso el impulso a vomitar, lo que marca un punto de inflexión notable con el grupo anterior. También resulta interesante el dato de una mayor variabilidad como grupo a la hora de indicar el PIA más molesto, si bien destaca el pensar que se está gordo/a (mientras se observan en el espejo, o simplemente, sin ningún elemento desencadenante), junto con la necesidad de hacer die- 
ta y el pensamiento de que los «demás» están más delgados que uno mismo. En comparación con el grupo SR, el grupo subclínico presentó una reacción emocional negativa más diversa y más frecuente, sobre-estimaron la amenaza de la intrusión y otorgaron un significado personal a la misma. En cuanto a las estrategias, las menos practicadas por el grupo subclínico fueron son las de los rituales no alimentarios, y las más utilizadas la distracción conductual y usar un contra-pensamiento.

Por último, en cuanto a las limitaciones del presente estudio, resaltar, en primer lugar, todas aquellas derivadas de la propia modalidad del instrumento utilizado: autoinforme, con lo que no se puede asegurar la ausencia de sesgos en cuanto a deseabilidad social, o accesibilidad y fidelidad de los recuerdos, entre otros (Gallego et al., 2007). Por otro lado, las reacciones emocionales, valoraciones disfuncionales, y estrategias de control se evalúan a través de una sola pregunta relacionada con el PIA más molesto. Los resultados de los análisis factoriales indican que esta segunda parte del INPIAS debe ser utilizada de forma sumativa, con lo que esta limitación quedaría, al menos en parte, atenuada. Finalmente, el INPIAS recoge las intrusiones desde un punto de vista de «producto», obviando todos los matices e información que se podrían capturar a través de otra metodología capaz de analizar las operaciones y procesos de la misma. Y, como es natural, la validez y utilidad clínicas del cuestionario deben ser objeto de un análisis específico más amplio, en el que se incluyan personas con diagnóstico de TA.

\section{REFERENCIAS}

Belloch, A., Cabedo, E., Morillo, C., Lucero, M., y Carrió, C. (2003). Diseño de un instrumento para evaluar las creencias disfuncionales del Trastorno Obsesivo Compulsivo: Resultados preliminares del Inventario de Creencias Obsesivas (ICO). Revista Internacional de Psicología Clínica y de la Salud, 3, 235-250.

Belloch, A., Morillo, C., Lucero, M., Cabedo, E., y Carrió, C. (2004). Intrusive thoughts in non-clinical subjects: the role of frequency and unpleasantness on appraisal ratings and control strategies. Clinical Psychology and Psychotherapy, 11, 100-110.
Belloch, A., Morillo, C., y García-Soriano, G. (2007). Are the dysfunctional beliefs that predict worry different from those that predict obsessions? Clinical Psychology and Psychotherapy, 14, 438-448.

Belloch, A., Morillo, C., y García-Soriano, G. (2006). Obsession subtypes: relationship with obsessive-compulsive symptoms, dysfunctional beliefs and thought control strategies. Revista de Psicopatología y Psicología Clínica, 11, 65-78.

Benedito, M.C., Perpiná, C., Botella, C., y Baños, R. (2003). Imagen corporal y restricción alimentaria en adolescentes. Anales de Pediatría, 58, 268-272.

Brown, T.A., Cash, T.F., y Mikulka, P.J. (1990). Attitudinal body image assessment: Factor analyses of the Body-Self Relations Questionnaire. Journal of Personality Assessment, 55, 134-155.

Bulik, C., Sullivan, P.F., Fear, J., y Pickering, A. (1997). Predictors of the development of bulimia nervosa in women with anorexia nervosa. Journal of Nervous and Mental Disease, 185, 704-707.

Cabedo, E., Belloch, A., Morillo, C., Giménez, A., y Carrió, C. (2004). Intensidad de las creencias disfuncionales en relación con el incremento en obsesividad. International Journal of Clinical and Health Psychology, 4, 465-479.

Carrasco, A (2006). Evaluación del perfeccionismo, la indecisión e intolerancia a la incertidumbre. Trabajo Investigación DEA. Universidad de Valencia.

Cattell, R.B. (1966). The scree test for the number of factors. Multivariate Behavioral Research, 1, 245-276.

Castro, J., Toro, J., Salamero, M., y Quimera, E. (1991). The Eating Attitudes Test: Validation of the Spanish version. Psychological Assessment, 7, 175-179.

Clark, D.A. (Ed). (2005). Intrusive thoughts in clinical disorders. Theory, research, and treatment. New York: Guildford Press.

Clark, D.A., y Beck, A.T. (2002). Manual for the Clark-Beck Obsessive Compulsive Inventory. Tejas. Psychological Corporation.

Clark, D.A., y Purdon, C. (1993). New perspectives for a cognitive theory of obsessions. Australian Psychologist, 28, 161-167.

Cohen, J. (1988). Statistical power analysis for the behavioral sciences $\left(2^{\text {nd }}\right.$ ed.) Hillsdale, NJ: Lawrence Erlbaum Associates.

Frost, R., Marten, P., Lahart, C., y Rosenblate, R. (1990). The dimensions of perfectionism. Cognitive Therapy \& Research, 14, 449-468.

Gallego, M.J., Botella, C., Quero, S., Baños, R.M., y García-Palacios, A. (2007). Propiedades psicométricas de la Escala de Miedo a la Evaluación Negativa (BFNE) en nuestra clínica. Revista de Psicopatlogía y Psicología Clínica, 12, 136-176.

García-Soriano, G. (2008). Pensamientos intrusos obsesivos y obsesiones clínicas: Contenidos y significado 
personal. Tesis Doctoral. Facultad de Psicología, Universidad de Valencia.

Garner D.M, y Garfinkel P.E. (1979). The Eating Attitudes Test: an index of the symptoms of anorexia nervosa. Psychological Medicine, 9, 273-279.

Garner, D.M., Olmsted, M.P., Bohr, Y., y Garfinkel, P.E. (1982). The eating attitudes test: Psychometric features and clinical correlates. Psychological Medicine, 12, 871-878.

Giménez, A., Morillo, C., Belloch, A., y Cabedo, E. (2004). Creencias disfuncionales asociadas a síntomas obsesivo-compulsivos: Una aproximación a la especificidad. Revista de Psicopatología y Psicología Clínica, 9, 1-17.

Heatherton, T.F., Herman, C.P., Polivy, J., King, G.A., y McGree, S.T. (1988). The (miss)measurement of restraint and analysis of conceptual and psychometric issues. Journal of abnormal Psychology, 97, 19-28.

Herman, C.P., y Polivy, J. (1980). Restrained eating. En A.J. Stunkard (Ed.), Obesity. Filadelfia, P.A: Saunders.

Herman, C.P., y Polivy J. (2007). Norm-violation, normadherence, and overeating. Collegium Antropologicum, 31, 55-62.

Humphreys, J.D., Clopton, J.R., y Reich, D.A. (2007). Disordered eating behaviour and obsessive compulsive symptoms in college students and affective similarities. Eating Disorders, 15, 247-259.

Joquera, M., Baños, R.M., Perpiñá, C., y Botella, C. (2005). La escala de estima corporal (BES): Validación en una muestra española. Revista de Psicopatología y Psicología Clínica, 10, 173-192.

Jones, C., Harris, G., y Leung., N. (2005). Parental rearing behaviours and eating disorders: The moderating role of core beliefs. Eating Behaviors, 6, 355-364.

Kline P., y Barret, P. (1983). The factors in personality questionnaires among normal subjects. Advances in Behaviour Research \& Therapy, 5, 141-202.

Meyer, T.J., Miller, M.L., Metzger, R.L., y Borkovec, T.D. (1990). Development and validation of the Penn State Worry Questionnaire. Behaviour Research and Therapy, 28, 487-495.

McKinley, N.M. (2002). Feminist perspectives and objectified body consciousness. En T.F. Cash y T. Pruzinsky (Eds.), Body Image. New York: Guilford Press.

Milos, G., Spindler, A., Ruggiero, G., Klaghofer, R., y Schnyder, U. (2002). Comorbidity of obsessive-compulsive disorders and duration of eating disorders. International Journal of Eating Disorders, 31, 284-289.

Purdon, C., y Clark, D.A. (1994). Obsessive intrusive thoughts in nonclinical subjects. Part II. Cognitive appraisal, emotional response and thought control strategies. Behaviour Research and Therapy, 32, 403-410.

Purdon, C., y Clark, D.A. (2001). Suppression of obsessionlike thoughts in non-clinical individuals: Impact on thought frequency, appraisal and mood state. Behaviour Research and Therapy, 39, 1163-1181.

Rachman, S., y de Silva, P. (1978). Abnormal and normal obsessions. Behaviour Research and Therapy, 16, 233248.

Ranson, K.M., Kaye, W.H., Weltzin, T.E., Rao, R., y Matsunaga, H. (1999). Obsessive-compulsive disorder symptoms before and after recovery from bulimia nervosa. American Journal Psychiatry, 156, 1703-1708.

Reina, N., Belloch, A., Morillo, C., y Clark, D. (2007). The Clark-Beck Obsessive-Compulsive Inventory: Validation of the Spanish version as a screening instrument for the obsessive-compulsive disorder. Poster V World Congress of Behavioral and Cognitive Therapies, Barcelona.

Sandín, B., y Chorot, P. (1991). Adaptación española del Penn State Worry Questionnaire. Madrid: UNED.

Shafran, R. (2002). Eating disorders and obsessive compulsive disorder. En Frost, R.O., y Steketee, G. (Ed.), Cognitive approaches to obsessions and compulsions: Theory, assessment, and treatment. Oxford: Pergamon.

Shafran, R., Bryant-Waugh, R., Lask, B., y Arscott, K. (1995). Obsessive compulsive symptoms in children with eating disorders: A preliminary investigation. $E a-$ ting Disorders: Journal of Treatment and Prevention, 3, 304-310.

Slade, P. D. (1982). Towards a functional analysis of anorexia nervosa and bulimia. British Journal of Clinical Psychology, 21, 167-179.

Serpell, L., Livingston, A., Neiderman, M., y Lask, B. (2002). Anorexia nervosa: Obsessive-compulsive disorder, obsessive-compulsive personality or neither? Clinical Psychology Review, 22, 647-669.

Sirton, M.M., y Birch, L.L. (2005). Weight status and psychosocial factors predict the emergence of dieting in preadolescent girls. International Journal of Eating Disorders, 38, 346-354.

Speranza, M., Corcos, M., Godart, N., Loas, G., Guilbau, O., Jeammet, P., y Flament, M. (2001). Obsessive compulsive disorders in eating disorders. Eating Behaviour, 2, 193-207.

Tanofsky-Kraff, M., Yanovski, S.Z., Wilfley, D.E., Marmarosh, C., Morgan, C.M., y Yanovski, J.A. (2004). Eating-disordered behaviors, body fat, and psychopathology in overweight and normal-weight children. Journal of Consulting and Clinical Psychology, 72, 53-61.

Thornton, C., y Russell, J. (1997). Obsessive compulsive comorbidity in the dieting disorders. International Journal of Eating Disorders, 21, 83-87.

Vartaniana, L.R., Herman, C.P., y Polivy, J. (2007). Consumption stereotypes and impression management: How you are what you eat. Appetite, 48, 265-277. 